\title{
An Implementation of Eye Movement-Driven Biometrics in Virtual Reality
}

\author{
Dillon Lohr \\ Department of Computer Science \\ Texas State University \\ dj170@txstate.edu
}

\author{
Samuel-Hunter Berndt \\ Department of Computer \\ Science and Engineering \\ Michigan State University \\ berndts2@msu.edu
}

\author{
Oleg Komogortsev \\ Department of Computer \\ Science and Engineering \\ Michigan State University \\ ok@msu.edu
}

\begin{abstract}
As eye tracking can reduce the computational burden of virtual reality devices through a technique known as foveated rendering, we believe not only that eye tracking will be implemented in all virtual reality devices, but that eye tracking biometrics will become the standard method of authentication in virtual reality. Thus, we have created a real-time eye movement-driven authentication system for virtual reality devices. In this work, we describe the architecture of the system and provide a specific implementation that is done using the FOVE head-mounted display. We end with an exploration into future topics of research to spur thought and discussion.
\end{abstract}

\section{CCS CONCEPTS}

-Security and privacy $\rightarrow$ Biometrics; $\bullet$ Human-centered computing $\rightarrow$ Virtual reality;

\section{KEYWORDS}

biometrics, eye tracking, virtual reality, authentication

\section{ACM Reference Format:}

Dillon Lohr, Samuel-Hunter Berndt, and Oleg Komogortsev. 2018. An Implementation of Eye Movement-Driven Biometrics in Virtual Reality. In ETRA '18: 2018 Symposium on Eye Tracking Research and Applications, June 14-17, 2018, Warsaw, Poland. ACM, New York, NY, USA, Article 4, 3 pages. https://doi.org/10.1145/3204493.3208333

\section{INTRODUCTION}

Biometrics is becoming a secure and accurate form of user authentication in the modern world. In 2014, over 200 million smart phones with fingerprint sensors were shipped worldwide. This number is expected to surpass one billion in $2018^{1}$. We believe that in the near future, a prevalent form of user authentication will be based on eye movements in virtual reality (VR) devices, especially those that are powered by non-image-based eye tracking sensors. VR has been successful due to its ability to obscure the difference between a user's perception of reality and created reality, allowing the user to act out situations that may otherwise be hard to replicate. Head-mounted displays (HMDs) are now equipped with gyroscopes,

\footnotetext{
${ }^{1}$ www.statista.com/statistics/522055/global-smartphone-fingerprint-shipments/

Permission to make digital or hard copies of part or all of this work for personal or classroom use is granted without fee provided that copies are not made or distributed for profit or commercial advantage and that copies bear this notice and the full citation on the first page. Copyrights for third-party components of this work must be honored.

For all other uses, contact the owner/author(s)

ETRA '18, June 14-17, 2018, Warsaw, Poland

(C) 2018 Copyright held by the owner/author(s)

ACM ISBN 978-1-4503-5706-7/18/06.

https://doi.org/10.1145/3204493.3208333
}

accelerometers, and IR motion tracking, allowing the application to mimic the user's real-world movements and thus making the user believe he/she is truly in the VR environment. In an effort to make VR more energy efficient through a concept known as foveated rendering [Guenter et al. 2012], eye tracking sensors have been installed in recently developed HMDs such as the FOVE and the HTC Vive (via Tobii Pro integration).

Using the recent integration of eye tracking in HMDs such as the FOVE, we've developed an application which employs eye movement-driven biometrics to authenticate users in a VR environment. This work was done in order to demonstrate a prototype of such a system.

\section{RELATED WORK}

A survey of eye movement-driven authentication was conducted where the authors tested the various methods currently being used for authentication, explored the many challenges involved in data collection, and proposed directions for future research and development [Galdi et al. 2016]. An important work recently defined a concept of temporal persistence in biometrics and indicated that it is possible to achieve an equal error rate (EER) of $2 \%$ with eye movement-driven biometrics during the task of reading while using high-quality eye movement data [Friedman et al. 2016].

In this work, we developed a VR implementation of the biometric framework created by Holland and Komogortsev [2014] with 3D gaze information. To the best of our knowledge, this is the first instance of implementing eye movement-driven biometrics in VR.

\section{METHOD}

To present stimuli in VR, we modified a C++/OpenGL project written by Dillon Lohr which uses Valve's OpenVR API to communicate with any compatible VR HMD via SteamVR.

The stimulus used comprises a white sphere with a smaller black sphere centered at its core. By rendering the black sphere after the white sphere and disabling depth testing, the black sphere is drawn over the white sphere. Thus, we mimic a standard target stimulus regardless of the angle between the user and the stimulus. Head position and orientation is ignored to keep the stimulus positioned correctly on the screen at all times.

We used the FOVE 0 VR HMD, which is capable of binocular eye tracking at $120 \mathrm{~Hz}$. Though, it should be noted that we requested a sample every $8 \mathrm{~ms}$ and received new samples at a rate of only $60 \mathrm{~Hz}$ (on average). The FOVE 0 houses a single display with a resolution of $2560 \times 1440$ pixels allowing up to a $100^{\circ}$ field of view ${ }^{2}$.

\footnotetext{
${ }^{2}$ https://www.getfove.com/
} 
Figure 1: Overview of the biometric authentication process.

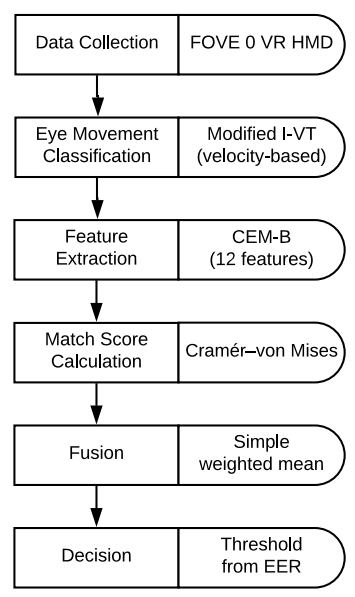

Since the FOVE calculates the 3D direction of the gaze (as opposed to the $2 \mathrm{D}$ point of regard that is normally calculated by eye trackers outside VR), we converted this direction, $\vec{g}$, to degrees of the visual angle, $\theta_{x}$ and $\theta_{y}$, using equations 1 and 2 respectively.

$$
\begin{aligned}
& \theta_{x}=\tan ^{-1}\left(g_{x} / g_{z}\right) \\
& \theta_{y}=\tan ^{-1}\left(g_{y} / g_{z}\right)
\end{aligned}
$$

Currently, the system is configured in a way to produce 100 hoirzontal saccades with amplitudes of 30 degrees of the visual angle ( \pm 15 degrees from the center). Each saccade is separated by a one-second-long fixation period. A user can perform an enrollment procedure, during which eye movement features are extracted and saved for future authentication purposes. The programmed system has the functionality to authenticate the current user against other enrolled users, including themselves.

Following the biometric framework from Holland and Komogortsev [2014], for each user, four fixation and eight saccade features are extracted from the signals for a total of twelve eye movement features. The fixation features are start time, duration, and centroid $(\mathrm{x}, \mathrm{y})$. For saccades, we determined the start time, duration, amplitude $(\mathrm{x}, \mathrm{y})$, mean velocity $(\mathrm{x}, \mathrm{y})$, and max velocity $(\mathrm{x}, \mathrm{y})$. The system is programmed to compare the feature distributions between two templates to get a vector of match scores, and these scores are combined into a single fused score using a simple weighted mean fusion with equal weights.

\section{CONCLUSION}

We implemented a biometric framework based on complex eyemovement behaviors in VR. Although previous work on eye movement biometrics commonly used eye trackers with very high sampling frequencies $(250+\mathrm{Hz})$ [Bednarik et al. 2005; Holland and Komogortsev 2013], we worked with only $60 \mathrm{~Hz}$ signals with nonuniform time intervals. The non-uniformity of the time intervals would have affected the start time and duration features for both fixations and saccades in the order of tens of milliseconds. Since the effect was random and present in all samples, we do not believe it would have an unfair impact on our results; however, it is a problem that should be thoroughly evaluated and corrected if necessary.
We believe that as VR technology becomes more mainstream, there will be a significant demand for hands-free authentication methods. Of those available, eye tracking especially has potential to become a stand-alone system or an important component of a multi-modal biometric system.

\section{CHALLENGES \& FUTURE WORK}

There are some notable challenges associated with eye movement biometrics in VR compared to a standard 2D paradigm. First, and perhaps most important, is the classification of 3D eye movements. There are several eye movement types to consider during classification, including: fixation, saccade, smooth pursuit, post-saccadic oscillation (glissade), vestibulo-ocular reflex, optokinetic nystagmus, and vergence. Improper identification of basic eye movement types can lead to various classification errors [Friedman et al. 2018] which subsequently could lead to poorly shaped feature distributions and inferior temporal precision of features. As such, no matter which classification algorithm is employed for the classification of various eye movement types, it is imperative that eye movements are accurately classified. The addition of depth to 3D stimuli can elicit vergence eye movement responses. These movements may be helpful in providing person-specific features. Second, research indicates that finding and employing temporally persistent features would most likely improve the biometric performance of the resulting features [Friedman et al. 2016]. Third, we noticed some calibration issues in the data we collected, despite using FOVE's built-in calibration procedure. A calibration routine could be prepended to each task in future research so that a regression model may correct for any discrepancies in the user calibration model. In fact, since HMDs are robust to slippage, such a calibration routine may only be necessary once every few tasks. The final challenge we will mention is the consideration of which stimulus and how many stimuli should be used for authentication such that it will ensure high accuracy while remaining feasible for the users of the system.

In the future, we intend to test the framework on a large and diverse subject pool so that we may produce statistically significant results. Additionally, we will compare different machine learning techniques such as a support vector machine (SVM) and K-means in place of simple weighted mean fusion to determine the optimal method for authentication. We are also interested in using a more extensive feature set as in Rigas et al. [2018] and introducing new, $3 \mathrm{D}$-specific eye movement features (especially vergence) to the biometric templates for enhanced security and to take advantage of the VR aspect of this research. In order to elicit vergence responses, we plan to modify the gaze stimulus to be more obviously 3D through the use of lighting and utilizing depth in the movement of the stimulus.

\section{ACKNOWLEDGEMENTS}

This work is supported by NSF grants CNS-1250718 and CNS1714623 and by Google Virtual Reality Research Awards bestowed on Dr. Komogortsev in years 2016 and 2017. 


\section{REFERENCES}

Roman Bednarik, Tomi Kinnunen, Andrei Mihaila, and Pasi Fränti. 2005. Eyemovements as a biometric. In Scandinavian conference on image analysis. Springer $780-789$.

Lee Friedman, Ioannis Rigas, Evgeny Abdulin, and Oleg Komogortsev. 2018. A Novel Evaluation of Two Related, and Two Independent Algorithms for Eye Movement Classification during Reading. Journal of Behavioral Research Methods (2018). In press.

Lee Friedman, Ioannis Rigas, Mark S Nixon, and Oleg V Komogortsev. 2016. Method to Assess the Temporal Persistence of Potential Biometric Features: Application to Oculomotor, and Gait-Related Databases. arXiv preprint arXiv:1609.03948 (2016).

Chiara Galdi, Michele Nappi, Daniel Riccio, and Harry Wechsler. 2016. Eye movement analysis for human authentication: a critical survey. Pattern Recognition Letters 84 (2016), 272-283.

Brian Guenter, Mark Finch, Steven Drucker, Desney Tan, and John Snyder. 2012 Foveated 3D Graphics. https://www.microsoft.com/en-us/research/publication/ foveated-3d-graphics/

Corey D. Holland and Oleg V Komogortsev. 2013. Complex Eye Movement Pattern Biometrics: The Effects of Environment and Stimulus. IEEE Transactions on Information Forensics and Security 8, 12 (Dec 2013), 2115-2126. https://doi.org/10.1109/ TIFS.2013.2285884

Corey D. Holland and Oleg V. Komogortsev. 2014. Software Framework for an Ocular Biometric System. In Proceedings of the Symposium on Eye Tracking Research and Applications (ETRA '14). ACM, New York, NY, USA, 365-366. https://doi.org/10. $1145 / 2578153.2582174$

Ioannis Rigas, Lee Friedman, and Oleg Komogortsev. 2018. Study of an Extensive Set of Eye Movement Features: Extraction Methods and Statistical Analysis. Fournal of Eye Movement Research 11, 1 (2018). https://bop.unibe.ch/JEMR/article/view/3795 